\title{
Unusual Thermal Diffusion in Polymer Solutions
}

\author{
Berend-Jan de Gans, ${ }^{1, *}$ Rio Kita, ${ }^{1, \dagger}$ Simone Wiegand, ${ }^{1,2, \ddagger}$ and Jutta Luettmer-Strathmann ${ }^{3, \S}$ \\ ${ }^{1}$ Max Planck Institut für Polymerforschung, Ackermannweg 10, D-55128 Mainz, Germany \\ ${ }^{2}$ Forschungszentrum Jülich GmbH, IFF-Weiche Materie, D-52428 Jülich, Germany \\ ${ }^{3}$ Department of Physics, University of Akron, Akron, Ohio 44325-4001, USA
}

(Received 10 August 2002; published 9 December 2003)

\begin{abstract}
Thermal diffusion forced Rayleigh scattering results on thermal diffusion of poly(ethylene oxide) (PEO) in ethanol/water mixtures are presented. In water-rich solvent mixtures, PEO is found to migrate towards regions of lower temperature. This is typical for polymer solutions and corresponds to a positive Soret coefficient of PEO. In solvent mixtures with low water content, however, the polymer is found to migrate towards higher temperatures, corresponding to a negative Soret coefficient of PEO in ethanol-rich solutions. To our knowledge, this is the first observed sign change of the Soret coefficient of a polymer in solution. We also present a simple lattice model for the polymer solvent system and calculate Soret coefficients with statistical mechanics methods. The calculated values agree qualitatively with the experimental results.
\end{abstract}

DOI: $10.1103 /$ PhysRevLett.91.245501

PACS numbers: $61.25 . \mathrm{Hq}, 61.43 . \mathrm{Bn}, 66.10 . \mathrm{Cb}$

A temperature gradient applied to a fluid mixture generally induces net mass flows which lead to the formation of concentration gradients. This effect is known as thermal diffusion or the Ludwig-Soret effect [1,2]. In the stationary state where the mass flows vanish, the magnitude of the effect is described by the Soret coefficients $S_{T, i}$ :

$$
S_{T, i}=-\frac{1}{c_{i 0}\left(1-c_{i 0}\right)} \frac{\nabla c_{i}}{\nabla T}
$$

where $c_{i}$ is the mass fraction of component $i, c_{i 0}$ is its equilibrium value, and where $T$ is the temperature. $S_{T, i}$ is positive if component $i$ moves to the low temperature region. Since the mass fractions add up to unity, $\sum_{i} c_{i}=1$, a $K$-component mixture has $K-1$ independent Soret coefficients.

Typically, the Soret coefficient of the heavier component of a binary liquid mixture is positive. This is not always the case, however, and the Soret coefficients in some low molecular weight liquid mixtures are known to change sign [2]. A sign change of the Soret coefficient was also observed in very recent thermophoresis experiments on protein solutions [3]. While there is no theory which reliably predicts the sign of the Soret effect in liquid mixtures, approaches based on the "heat of transfer" concept [2] suggest that molecular interactions play an important role. In recent experiments, Debuschewitz and Köhler [4] identified two distinct contributions to the Soret coefficients of isotope substituted liquid mixtures of benzene and cyclohexane. The first contribution, due to differences in the molecules' masses and moments of inertia, was found to be independent of composition of the mixture. The second, reflecting chemical differences of the molecules, was found to vary with composition and change sign, inducing a sign change of the total Soret coefficients.
With only two known exceptions, polymers in solution conform to the rule that the heavier component migrates to the colder regions of the fluid. Giglio and Vendramini [5] found a negative Soret coefficient for poly(vinyl alcohol) in water. Very recently, we reported our first thermal diffusion results for poly(ethylene oxide) (PEO) in ethanol-rich ethanol/water mixtures which indicated that the polymer migrates to the warmer region in these mixed solvents [6]. In this work, we investigate systematically the Soret effect of PEO in ethanol/water mixtures. We expect interesting thermal diffusion properties for this system, since the interactions between the polymer and the two solvents are very different. Hydrogen bonding makes water an excellent solvent for PEO at room temperature while PEO is insoluble in ethanol. Because of the biocompatibility of PEO, this system is also relevant for biological applications (cf. [7]).

Experiment. - We measured Soret coefficients with a holographic grating technique called thermal diffusion forced Rayleigh scattering (TDFRS) with heterodyne detection and active phase tracking [8]. The principle of TDFRS is analogous to ordinary forced Rayleigh scattering: An intensity grating is created by the interference of two laser beams. A trace amount of inert absorbing dye added to the sample converts the intensity grating within microseconds into a temperature grating, which is converted into a concentration grating within milliseconds by the effect of thermal diffusion. Both temperature and composition grating contribute to a refractive index grating readout by Bragg diffraction of a third laser beam.

For a binary mixture, the heterodyne signal intensity reflects a single concentration mode whose time constant and amplitude are related to the mass-diffusion and Soret coefficient of the mixture, respectively [8]. For a polymer in a mixed solvent, there are two concentration modes with widely separated time constants. The normalized 
heterodyne signal intensity $\zeta_{\text {het }}(t)$ for the time after the intensity grating has been switched on is derived for the PEO/ethanol/water system in Ref. [9] and given by

$$
\begin{aligned}
\zeta_{\text {het }}(t)=1+\sum_{i=0,1} & \left(\frac{\partial n}{\partial T}\right)_{i}^{-1}\left(\frac{\partial n}{\partial c}\right)_{i} S_{T i} c_{i}\left(1-c_{i}\right) \\
& \times\left(1-e^{-q^{2} D_{i} t}\right),
\end{aligned}
$$

where $t$ is the time, $n$ is the index of refraction, and $q$ is the grating wave number. $D_{i}$ and $S_{T, i}, i=0,1$, denote the mass-diffusion and Soret coefficients, respectively, where $i=0$ refers to the polymer in the mixed solvent while $i=1$ refers to the solvent mixture. The contrast factors multiplying the exponential terms contain derivatives of the index of refraction, which are determined independently with a Michelson interferometer operating at a wavelength of $632.8 \mathrm{~nm}$. Hence, mass-diffusion and Soret coefficients of the ternary PEO/ethanol/water mixtures can be determined from the time constants and amplitudes of the normalized heterodyne signal.

The PEO in our experiments had a molecular weight of $M_{\mathrm{w}}=2.65 \times 10^{5} \mathrm{~g} \mathrm{~mol}^{-1}$ with a polydispersity PD = 1.1. All samples were semidilute solutions containing $5.0 \pm 0.1 \mathrm{~g} \mathrm{~L}^{-1}$ PEO. Fifteen different solvent mixtures were studied, the water content varying between $5 \%$ and $100 \%$ by weight. A trace amount of dye (quinizarin, Sigma-Aldrich) was added to the samples (optical density $1-2 \mathrm{~cm}^{-1}$ ). As for PEO in pure water basantolyellow 215 (BASF) was used. Adsorption of the dyes on PEO does not occur as their absorption spectrum remains unchanged $[6,9]$.

Figure 1 shows typical normalized heterodyne signals as a function of time obtained for three different solvent compositions. The inset shows the two clearly separated decays for the $50 \mathrm{wt} \%$ mixture on a logarithmic time scale. Analysis of the amplitude and the decay time of the fast mode leads to the same values for the Soret coefficient of water as obtained from measurements of binary ethanol/water mixtures [9]. This confirms that the fast mode reflects the thermal diffusive behavior of the binary solvent mixture while the slow mode corresponds to the establishment of a concentration gradient of PEO in the solvent mixture. For long times, the heterodyne signals in Fig. 1 reflect the slow mode, which is seen to decrease with time for solvent compositions of $15.02 \mathrm{wt} \%$ and $50 \mathrm{wt} \%$ water. This indicates a negative Soret coefficient of PEO in mixtures with low water content. In contrast, the signal of PEO in pure water shows an increase with time, corresponding to a positive Soret coefficient of PEO in water. Figure 2 shows the Soret coefficient of PEO in ethanol/water as a function of water weight fraction. We observe a sign change of $S_{T}$ of PEO in ethanol/water mixtures at a weight fraction of $83 \%$. To our knowledge, this is the first observation of a sign change of the Soret coefficient of a polymer in solution. In pure ethanol/water mixtures with up to $25 \mathrm{wt} \%$ water, the signal contribution of the Soret effect is negligible compared to the thermal

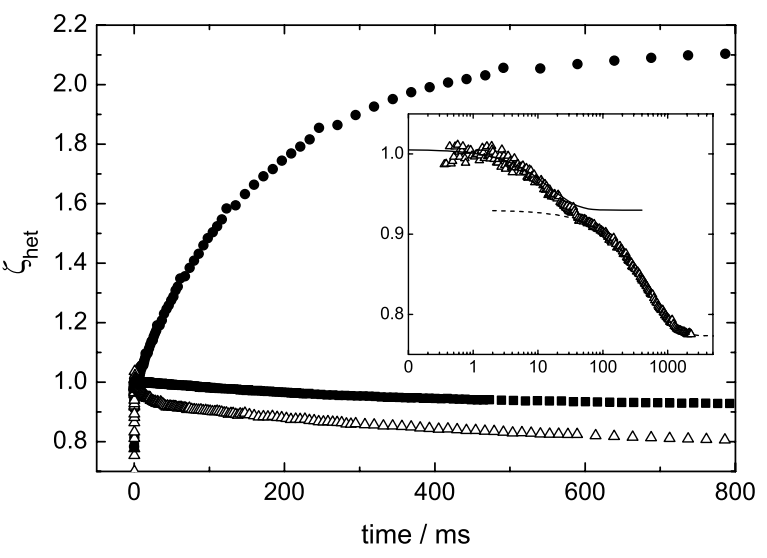

FIG. 1. Typical normalized heterodyne diffraction signal for a solution of PEO in ethanol/water $\left[15.02 \mathrm{wt} \% \mathrm{H}_{2} \mathrm{O}(\boldsymbol{\square})\right.$, $50 \mathrm{wt} \%(\triangle), 100 \mathrm{wt} \%(\bigcirc)]$. The inset shows the two decays for 50 wt $\%$ mixture ethanol/water versus logarithm of the time.

contribution [6,10]. Neglecting the solvent contribution to the thermal diffusion leads to $6 \%-10 \%$ smaller values for $S_{T}$ of PEO in ethanol/water mixtures, which is just barely outside the error bars [6]. In the middle concentration range, the influence of the solvent mixture is much stronger and the deviations exceed 30\% and more [9].

Theory. - In order to investigate the origin of the unusual experimental findings, we have developed a simple lattice model for a polymer chain in a mixed, compressible solvent [11]. We consider a simple cubic lattice of $N$ sites. The polymer chain occupies $N_{0}$ contiguous sites, while the two types of solvent particles occupy $N_{1}$ and $N_{2}$ single sites, respectively, and $N_{3}$ sites remain unoccupied, $N=\sum_{i=0}^{3} N_{i}$. Interactions between occupied nearestneighbor sites are described by interaction energies $\epsilon_{i j}$, $i, j \in\{0,1,2\}$. In aqueous solutions, hydrogen bonding between PEO and water plays an important role (cf. [12]). In order to account for these specific interactions, each elementary cube representing water is assumed to have one special face. If this face is exposed to a polymer segment, the interaction energy is $\epsilon_{01 ; s}$ (strongly attractive); otherwise it is $\epsilon_{01 ; n}$ (nonspecific).

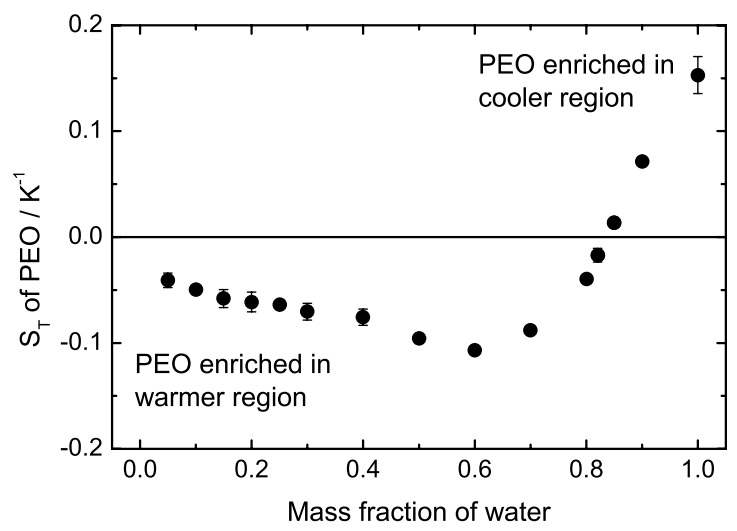

FIG. 2. Soret coefficient $S_{T}$ of PEO in ethanol/water as a function of water weight fraction. 
From an exact enumeration of all self-avoiding random walks of length $N_{0}-1$ on a simple cubic lattice (cf. [13]), we determine the number $c(m)$ of chain conformations with $m$ segment pair contacts and the average chain dimensions as a function of $m$. In this work $N_{0}=17$. Under a random mixing approximation for all but the polymer contacts, the canonical partition function for the system can be written as

$$
\begin{aligned}
Z= & N \sum_{m} c(m) \sum_{\left[n_{1}\right]} 6^{N_{1}-n_{1}}\left(\begin{array}{c}
n_{n} \\
n_{1}
\end{array}\right)\left(\begin{array}{c}
N-n_{n}-N_{0} \\
N_{1}-n_{1}
\end{array}\right) \times \sum_{\left[n_{2}\right]}\left(\begin{array}{c}
n_{n}-n_{1} \\
n_{2}
\end{array}\right)\left(\begin{array}{c}
N-n_{n}-N_{0}-\left(N_{1}-n_{1}\right) \\
N_{2}-n_{2}
\end{array}\right) \\
& \times e^{-\beta\left(m \epsilon_{00}+n_{2} \epsilon_{02}\right)}\left(5 e^{-\beta \epsilon_{01 ; n}}+e^{\left.-\beta \epsilon_{01 ; s}\right)^{n_{1}}} e^{-\beta E_{r}},\right.
\end{aligned}
$$

where $\beta=1 / k_{\mathrm{B}} T, k_{\mathrm{B}}$ is Boltzmann's constant, $n_{n}=$ $4 N_{0}+2-2 m$ is the number of nearest-neighbor sites of the polymer which are occupied by $n_{i}, i \in\{1,2,3\}$, solvent particles and voids. The square brackets around the summation indices indicate that the summation is performed consistent with the available nearest-neighbor sites and the total filling of the lattice. The energy $E_{r}$ denotes the contribution to the total energy due to solventsolvent interactions evaluated in random mixing approximation [14]. In the absence of the polymer, $Z$ reduces to the lattice fluid partition function of a compressible binary mixture of single site particles [14]. By performing partial summations over the terms in Eq. (3), we compute the average radius of gyration $R_{g}^{2}$ of the chain, which is used to characterize solvent quality (see Fig. 3). The pressure $P$ of the system is calculated from $\beta v P=$ $(\partial \ln Z / \partial N)_{N_{i, i} \neq 3}$, where $v$ is the volume of one lattice site. In this work, we retain the system-dependent parameters determined in Ref. [11] except for $\epsilon_{\mathrm{ws}}$, the energy of ethanol-water interactions. Originally, $\epsilon_{\mathrm{ws}}$ was estimated from the geometric mean approximation. Here, we determined a value of $\epsilon_{\mathrm{ws}}=-3600 \mathrm{~J} / \mathrm{mol}$ from a comparison with tabulated values for the density of ethanol-water mixtures [16], weighted to ensure a good

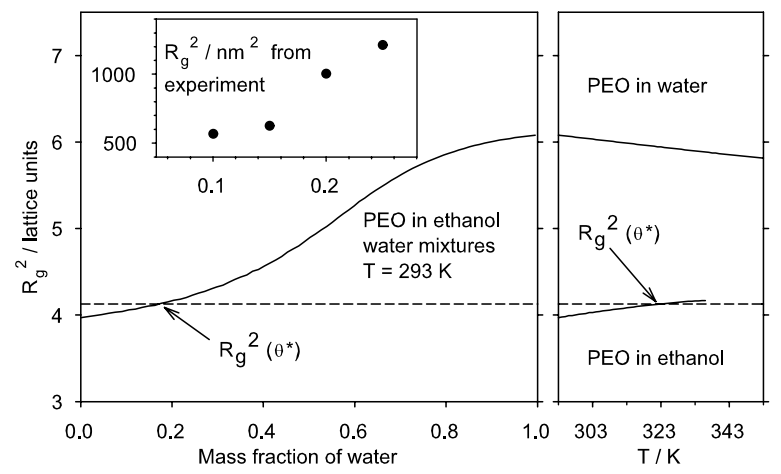

FIG. 3. Radius of gyration squared, $R_{g}^{2}$, of short PEO chains in ethanol/water mixtures. The left panel shows calculated chain dimensions as a function of solvent composition at temperature $T=293 \mathrm{~K}$, pressure $P \approx 0.1 \mathrm{MPa}$, and a PEO concentration of $5 \mathrm{~g} \mathrm{~L}^{-1}$. Experimental data from static light scattering [6] are presented in the inset. The right panel shows the temperature variation at constant pressure of the calculated chain dimensions of PEO in the two pure solvents ethanol and water. The dashed line indicates the chain dimensions, $R_{g}^{2}\left(\theta^{*}\right)$ of the isolated 17 bead chain at the $\theta$ temperature of the infinite chain [15]. fit at high water concentrations. In Fig. 3 we present graphs for the radius of gyration of the PEO chain. The chain expands (solvent quality improves) with increasing water content of the solution, in qualitative agreement with the experimental data [6] presented in the inset. For PEO in ethanol, the chain dimensions increase with increasing temperature while they decrease with temperature for PEO in water, in agreement with observed changes in solvent quality cf. Ref. [7].

In order to investigate the Soret effect, we consider a system divided into two chambers of equal size that are maintained at slightly different temperatures [11]. Particles are free to move between the chambers, which do not otherwise interact. If the pressure difference between the chambers is small enough to be neglected, the Soret coefficient can be determined from the difference in composition of the solutions in the two chambers [1]. Consider a single-chain system that is divided into two chambers, $A$ and $B$, with slightly different temperatures, $T^{A}>T^{B}$. Under the assumption that the chambers are noninteracting, the partition function of the whole system is a product of the partition functions of the individual chambers, $Z^{A} Z^{B}$. The chambers are represented by lattices with $N^{a}, a \in\{A, B\}$ sites and occupation numbers $N_{i}^{a}$, $i \in\{0, \ldots, 3\}$, where $N=N^{A}+N^{B}$ and $N_{i}^{B}=N_{i}-N_{i}^{A}$. The temperature difference employed here, $\delta T=$ $10^{-4} \mathrm{~K}$, is sufficiently small to neglect thermal expansion and we set $N^{A}=N^{B}=N / 2$. If the particles are allowed to move freely between the chambers, the sum $Q$ of all two-box configurations is given by

$$
Q=\sum_{\left[N_{i}^{A}\right]} Z^{A}\left(\left\{N_{i}^{A}\right\}\right) Z^{B}\left(\left\{N_{i}-N_{i}^{A}\right\}\right) \equiv Q_{0, A}+Q_{0, B},
$$

where the square brackets have the same meaning as previously. $Q_{0, A}$ and $Q_{0, B}$ represent the sums of states with the polymer in chambers $A$ and $B$, respectively. The fraction $Q_{0, A} / Q$ is the probability for finding the polymer in chamber $A$. A Taylor expansion shows that, to first order in the temperature difference $\delta T$, this probability is determined by a difference in internal energy

$$
Q_{0, A}-\frac{1}{2} \simeq-\frac{1}{4} \frac{\left\langle U_{\mathrm{nop}}\right\rangle-\left\langle U_{\mathrm{pol}}\right\rangle}{k_{\mathrm{B}} T} \frac{\delta T}{T} .
$$

Here $\left\langle U_{\text {nop }}\right\rangle$ and $\left\langle U_{\text {pol }}\right\rangle$ are the internal energies of two chambers at the same temperature, averaged over all 


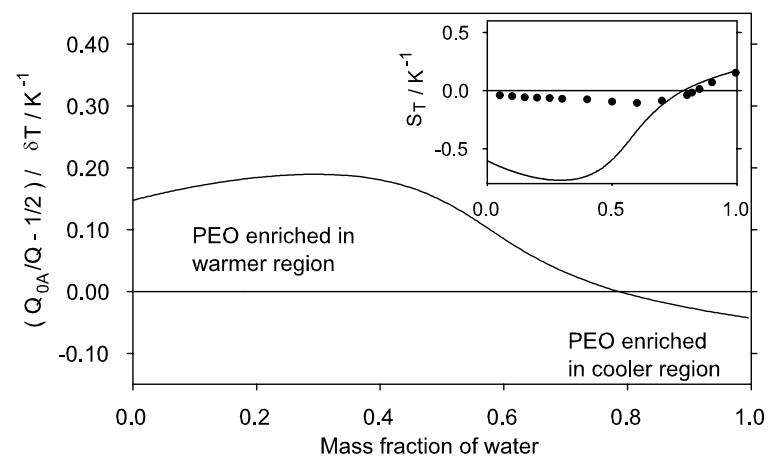

FIG. 4. Excess probability $Q_{0, A} / Q-1 / 2$ to find the polymer in the higher-temperature chamber as a function of water content of the solution for $T=293 \mathrm{~K}, \delta T=10^{-4} \mathrm{~K}$. The inset shows the lattice model predictions for the Soret coefficient and the experimental data reported in this work.

configurations of particles, where the polymer is confined to one of the chambers. Results for $Q_{0, A} / Q-1 / 2$ calculated from the sum of states are presented in Fig. 4. For low water concentrations, the polymer is more likely to be found in the higher temperature chamber while the opposite is true for high water concentrations. The inset of the figure shows lattice model estimates, $S_{T, \text { Lattice }}$, for the Soret coefficients of PEO obtained by monitoring the composition of each chamber during calculation of the sum of states, Eq. (4). Our simple lattice model, which takes only conformational contributions to thermodiffusion into account, uses short chains with parameters to emulate large chains, and neglects hydrogen bonding between solvent molecules, cannot be expected to yield quantitatively correct results. However, the calculated values for $S_{T, \text { Lattice }}$ initially decrease with increasing water content of the solvent, go through a minimum, and exhibit a change in sign of the Soret coefficient at high water content of the solution, in qualitative agreement with the experimental data.

Discussion. - Our experimental and theoretical investigation of PEO in ethanol/water mixtures gives some insight into conditions that lead to unusual thermodiffusion in polymer solutions. The experimental data presented in Figs. 1 and 2 and the calculated results presented in Figs. 3 and 4 illustrate the role of solvent quality. Positive Soret coefficients are observed for PEO in pure water, which is an excellent solvent, while negative Soret coefficients are observed for low water content of the solution, that is under poorer solvent conditions. The negative Soret coefficient reported [5] for poly(vinyl alcohol) in water at $25^{\circ} \mathrm{C}$ agrees with our findings since the system is very close to poor solvent conditions [17]. Our investigation leads us to expect other systems to yield changes in the sign of polymer Soret coefficients. For example, a solution of a copolymer in a single solvent may change sign as a function of composition of the copolymer, if the chains are composed of two types of segments, one with highly attractive and one with net repulsive segment-solvent interactions. For the PEO/ ethanol/water system studied here, hydrogen bonding plays a most important role. The Soret coefficient of PEO changes sign at a solvent composition, where large structural changes occur in binary mixtures of ethanol and water [18]. We are currently investigating this aspect in more detail.

The authors would like to thank Cindy Leppla, Thomas Wagner, Petra Räder, Beate Müller, and Christine Rosenauer for assistance with the experiments and Florian Müller-Plathe and Mark Taylor for helpful discussions. Financial support through the National Science Foundation (DMR-0103704), the Ohio Board of Regents (R5413), the Petroleum Research Fund (36559GB7), and the Research Corporation (CC5228) is gratefully acknowledged.

*Electronic address: bjdegans@gmx.de

${ }^{\dagger}$ Electronic address: kita@mpip-mainz.mpg.de

‡Electronic address: s.wiegand@fz-juelich.de

${ }^{\S}$ Electronic address: jutta@physics.uakron.edu

[1] S. deGroot and P. Mazur, Non-equilibrium Thermodynamics (Dover, New York, 1984).

[2] H. Tyrell, Diffusion and Heat Flow in Liquids (Butterworth, London, 1961).

[3] S. Iacopini and R. Piazza, Europhys. Lett. 63, 247 (2003).

[4] C. Debuschewitz and W. Köhler, Phys. Rev. Lett. 87, 055901 (2001).

[5] M. Giglio and A. Vendramini, Phys. Rev. Lett. 38, 26 (1977).

[6] B.-J. de Gans, R. Kita, B. Müller, and S. Wiegand, J. Chem. Phys. 118, 8073 (2003).

[7] E. E. Dormidontova, Macromolecules 35, 987 (2002).

[8] W. Köhler and R. Schäfer, Adv. Polym. Sci. 151, 1 (2000).

[9] R. Kita, S. Wiegand, and J. Luettmer-Strathmann (to be published).

[10] N. Arnaud and J. Georges, Spectrochim. Acta A 57, 1295 (2001).

[11] J. Luettmer-Strathmann, J. Chem. Phys. 119, 2892 (2003).

[12] C. Jeppesen and K. Kremer, Europhys. Lett. 34, 563 (1996).

[13] A. M. Nemirovsky, K F. Freed, T. Ishinabe, and J. F. Douglas, Phys. Lett. A 162, 469 (1992).

[14] S. M. Lambert, Y. Song, and J. M. Prausnitz, in Equations of State for Fluids and Fluid Mixtures, edited by J.V. Sengers, R. F. Kayser, C. J. Peters, and H. J. White, Jr. (Elsevier, Amsterdam, 2000), Chap. 14, pp. 523-588.

[15] H. Meirovitch and H. A. Lim, J. Chem. Phys. 92, 5144 (1990).

[16] Handbook of Chemistry and Physics, edited by R. C. West (Chemical Rubber, Boca Raton, FL, 1978), 59th ed.

[17] D. Eagland and N. J. Crowther, Faraday Symp. Chem. Soc. 17, 141 (1982).

[18] V.P. Belousov and M.Y. Panov, Thermodynamic Properties of Aqueous Solutions of Organic Substances (CRC Press, Boca Raton, FL, 1994). 